\title{
Carnets
}

Revue électronique d'études françaises de l'APEF

Deuxième série - 9 | 2017

Reconnaissances et légitimité en français

\section{Querelles et légitimations}

Quand le spectre de la mort de la littérature hante les débats

\section{Alain Viala}

\section{(2) OpenEdition}

Journals

Édition électronique

URL : http://journals.openedition.org/carnets/1995

DOI : $10.4000 /$ carnets. 1995

ISSN : 1646-7698

Éditeur

APEF

Référence électronique

Alain Viala, «Querelles et légitimations », Carnets [En ligne], Deuxième série - 9 | 2017, mis en ligne le

31 janvier 2017, consulté le 23 avril 2019. URL : http://journals.openedition.org/carnets/1995 ; DOI :

10.4000/carnets. 1995

Ce document a été généré automatiquement le 23 avril 2019

\section{(c) (i) \&}

Carnets est mis à disposition selon les termes de la licence Creative Commons - Atribution - Pas d'utilisation commerciale 4.0 International. 


\title{
Querelles et légitimations
}

\author{
Quand le spectre de la mort de la littérature hante les débats
}

\section{Alain Viala}

1 Je commencerai par évoquer deux brèves histoires de querelles littéraires. Parce que les querelles sont des situations de crise, et donc de bons révélateurs de certaines problématiques. Comme, ici, celle de la légitimité - que j'aborde du point de vue littéraire mais sans en négliger, au contraire, les enjeux sociaux et politiques.

2 Et je ferai suivre ces deux récits par trois questions et quatre réflexions sur cette problématique de la légitimité ou, plus exactement dit, de la légitimation.

Mon premier petit récit est une vieille histoire, qui remonte à 1738 et, qui, je pense, n'est pas très connue. Cette année-là, au mois de mars, un académicien, l'abbé d'olivet, publie des Remarques de grammaire sur Racine ${ }^{1}$. Il s'agit d'un commentaire stylistique des tragédies raciniennes, plus précisément (je cite sa page III) d'un inventaire des «petites fautes de style où elles peuvent être tombées ». Voilà donc un sage travail d'érudit plongé dans la philologie. Mais aussitôt, un autre homme de Lettres, l'abbé Desfontaines, l'attaque avec hargne, et, après une série d'échanges et de manœuvres ${ }^{2}$, il publie un volume où il contredit point par point les Remarques de d'olivet, et il lui donne un titre sensationnaliste : Racine vengé. Voilà donc la philologie considérée comme une vendetta. En apparence, ce n'est qu'une simple querelle de gens de Lettres parmi tant d'autres, y compris dans cette alacrité agressive de langage ${ }^{3}$. Mais sortir du fond des fagots un cas d'érudition que plus personne ne connaît aujourd'hui a une raison d'être parce que, sous ses dehors anecdotiques, cette affaire avait des enjeux pas si petits.

4 En effet, les Remarques de d'Olivet constituaient le lancement d'un projet bien plus vaste, celui de doter « la France de ses auteurs classiques ». «Classiques » s'entend ici au sens premier du terme, c'est-à-dire comme les auteurs dignes d'être enseignés dans les classes parce que « ils peuvent servir de modèles et pour bien penser et pour bien écrire ». Pour cela, d'olivet propose que soit programmée, sous le contrôle de l'Académie, une série d'éditions critiques des «ouvrages dont le mérite est depuis cinquante ou soixante ans avoué de tout le monde $»^{4}$. Et ses Remarques sur Racine rassemblent le matériau d'une édition critique exemplaire qui amorcerait la série. Face à quoi, Desfontaines ne conteste pas l'idée des classiques français mais taxe d'olivet d'une triple faute. Une faute de 
principe parce qu'il propose un modèle poétique quand les jeunes gens qui ont besoin d'apprendre à écrire doivent écrire en prose. Une faute de compétences, parce qu'il voit des erreurs de langue là où il s'agit en fait de licences poétiques, et puis un péché d'orgueil, parce qu'il s'arroge le droit de juger Racine. D'où l'affirmation que son livre est «dangereux pour les libertés du Parnasse Français », que Desfontaines veut, lui, sauvegarder.

On a donc là, à la fois, l'acte de naissance des classiques français, et, illico, un conflit sur les valeurs littéraires qu'ils incarnent. De la légitimation du littéraire aux débats sur l'institution de la littérature légitime, j'ai, pour ma part, vu là une suite des évolutions des données dont j'avais essayé d'analyser la structure dans un livre intitulé Naissance de l'écrivain ${ }^{5}$.

6 Mais puisque je viens de parler de la naissance de la littérature canonique en français, je passe à mon second récit, qui sera celui d'une chronique de leur mort annoncée.

7 L'affaire est beaucoup plus récente et certains d'entre vous en ont sans doute entendu parler. Elle date de l'an 2000. Le 4 mars de cette année-là le journal Le Monde publie deux tribunes libres, l'une signée par un groupe d'universitaires et intitulée «C'est la littérature qu'on assassine ! », et l'autre " Contre la suppression de la dissertation » signée de deux professeurs de lycée. Elles ont suscité des répliques immédiates, dès le 8 mars, par les dirigeants de l'Association Française de enseignants de français ${ }^{6}$ (on trouvera en annexe ces textes, du moins de larges extraits). Et puis de réponses aux réponses, d'articles en numéros de revue, et d'émissions de radio en meetings houleux à la Sorbonne, les débats se sont poursuivis durant quelques années, en un long collier de discours. Que je ne vais pas commenter très en détail, pas plus que les épisodes de la querelle, car ce serait trop long et puis il se trouve que j'ai été mêlé à cette querelle et je me dois de me comporter, comme le recommandait l'abbé Desfontaines, en "galant homme » et donc de ne pas en rajouter. Je précise donc seulement que la dispute portait cette fois sur les programmes d'enseignement et qu'elle opposait, d'un côté les partisans d'un enseignement centré sur la littérature patrimoniale et la dissertation, qui ont été désignés comme les «Républicains » et qui craignaient que les nouveaux programmes ne portassent tort à l'une et l'autre, et de l'autre côté, les partisans d'un enseignement rénové, qui ont été désignés comme des "Pédagogues». Autre version donc des débats sur la défense et illustration de la culture des classiques, tels que d'olivet les avait inventés (Pour ceux qui se sentiraient inquiets de la mort des classiques : les programmes ont été appliqués et les classiques en sont pas morts).

Voilà donc deux querelles. De l'une à l'autre, il y a des différences manifestes. Ne serait-ce que parce que l'une se situe au moment où l'enseignement de la littérature française commençait à peine, et l'autre au moment où tous les jeunes français vont à l'École et reçoivent tous un large enseignement littéraire. J'aurai à revenir sur ces différences. Mais si j'ai ainsi aligné ces deux épisodes, c'est parce que ce sont deux moments d'une même histoire, celle de la légitimation de la littérature. Et c'est aussi parce que, si l'on fait comme d'Olivet une étude de style, on voit qu'en 1738, il est proclamé de le Parnasse est en danger, qu'il faut le « conserver » et « venger » les poètes mal traités, et qu'en 2000 il est question de "péril ", de "trahison ", et qu'on se jette même à la tête des accusations réciproques d'« assassinat " de la littérature. Même vocabulaire tragique donc, de sorte que de l'un à l'autre de ces maillons de la chaîne historique de la littérature canonique en France affleurent des enjeux qui semblent tenir au plus profond du cœur ou de l'esprit des belligérants qui s'affrontent ainsi et puisqu'ils se vouent aux gémonies au nom de la 
culture légitime, de ses pompes et de ses œuvres et qui, par conséquent, touchent peutêtre en fait à des enjeux d'un autre ordre, plus collectif, voir identitaire.

La littérature légitime, ses pompes et ses œuvres seront donc les trois questions que je tenterai maintenant d'envisager à partir de ces deux histoires de querelles - à partir de, c'est-à-dire : en élargissant peu à peu la perspective, dans une démarche inductive.

Première question, l'objet même de la querelle, la littérature légitime et sa définition. Sur ce sujet, il semble qu'en 1738 comme en l'an 2002, la base soit un consensus omnium, que les auteurs canoniques sont ceux qui ont été, comme dit l'abbé d'olivet, «avoués par tout le monde ».

11 Mais le consensus omnium, on le sait bien, s'il peut tout justifier ne définit jamais rien, et sous ce consensus apparent se révèlent plusieurs strates de conflits, au moins trois. La première concerne l'extension même de ce corpus. En 1738, d'olivet s'intéresse aux poètes, tandis que Desfontaines le met au défi de faire la même chose (voyez la note 2) pour des prosateurs tels que « Pellisson, Fléchier, Bussy, Bouhours, Fleury et Vertot ». En 2000, l'AFEF revendique (voir annexe c) « un corpus littéraire considérablement élargi » et « la légitimité du recours aux littératures contemporaines et aux littératures en train de se faire ». Il dispute porte donc en fait sur la taille du Panthéon littéraire et l'abondance de la population qui peut y entrer.

12 Ce qui se trouve redoublé par une question qui touche au prix d'entrée dans ce Panthéon. En 1738, l'abbé Desfontaines épingle l'abbé d'olivet parce qu'il a exclu de l'œuvre de Racine la toute première tragédie de celui-ci, $L a$ Thébaïde, qu'il considérait comme un simple "essai d'un jeune homme », autant dire une erreur de jeunesse. La question qui apparaît là est celle de la modélisation, c'est-à-dire du tri entre les œuvres, tri qui peut s'accompagner parfois d'un travail pour les expurger, voire les corriger (comme le faisait d'olivet). De sorte que la culture légitimée n'apparaît pas comme une culture historiquement « vraie » (empiriquement conforme à ce que les auteurs ont produit) mais comme le point terminal d'un processus d'arrangements. Et de sorte qu'au total, la légitimation suppose non seulement une sélection des auteurs, mais aussi un formatage de leurs écrits. Alors la consécration se paye au prix d'une déformation. Donc au prix de la vérité historique. Et s'ajoute à cela une troisième strate de divergences, celle qui tient au fait que le corpus change forcément au fil du temps, parce la langue change, parce que de nouveaux auteurs sont reconnus, et parce que aussi, même si personne n'y prête attention, quand des nouveaux font leur entrée dans le corpus canonique, d'autres en sortent. Ainsi, Desfontaines mentionnait comme des exemples évidents les noms de Pellisson et Fléchier, Bouhours et Bussy, et plus encore Vertot et Fleury, qu'aujourd'hui plus personne ne connaît - c'est-à-dire pratique réellement - en dehors de quelques spécialistes. Bref, même si la consécration conserve, il existe de fait des dates de péremption.

13 Le consensus sur la littérature légitime est donc un trompe-l'œil. Ce dont, il faut le remarquer, les polémistes s'accommodent en ne livrant jamais leur liste d'auteurs canoniques - ce qui est pourtant faisable : je parle d'expérience, je m'y suis essayé (Viala, 2009) - et en jouant toujours sur l'implicite. Ils citent quelques noms, et font ainsi entendre une sorte de "vous voyez bien de quoi il s'agit» qui relève d'une logique ectoplasmique puisque les lecteurs sont ainsi invités à continuer eux-mêmes la liste. Il est 
vrai que ce procédé qui donne l'illusion d'un accord solide alors qu'il repose en fait sur une connivence labile est d'une efficacité redoutable ; au fond, c'est lui qui permet que les querelleurs puissent se quereller ...

Mais la connivence vole en éclats dès lors qu'on en vient à la question que j'appelais celle des "pompes » du canon, c'est-à-dire des usages et rituels auxquels il est voué, la question, pour le dire en termes simples, du " pour quoi faire? ». Là encore, on retrouve un consensus apparent : tout le monde affirme que le but est de donner « des modèles et pour bien penser et pour bien s'exprimer » - je cite d'olivet mais les mêmes termes reviennent dans les débats de l'an 2000, voyez encore en annexe). Mais quand on passe aux usages effectifs, le dissensus surgit. Ainsi en 1738, d'olivet imagine des classiques qui seront des modèles pour "les jeunes poètes »: alors, classiciser des poètes passés à l'usage des poètes futurs, c'est une logique de la reproduction. Pour Desfontaines, en revanche, comme les apprentis scripteurs auront à écrire en prose et que la poésie n'est qu'une pratique particulière et qui d'ailleurs ne s'enseigne pas en tant que telle, les modèles communs sont à chercher du côté des prosateurs. Et en l'an 2000, même si les données ont changé, on voit que les « Républicains » défendent - en particulier l'exercice de la dissertation tandis que les « rénovateurs » dénoncent «la crispation sur l'écriture d'interprétation et de commentaire» en soulignant qu' "on ne pratique pas la dissertation dans la vie » et que donc il faut former à d'autres types d'écrits. Et là encore resurgit la question de la "reproduction ", le même texte posant crûment la question :

Doit-on définir le programme d'apprentissage culturel de toute une classe d'âge en fonction des perspectives de spécialisation de quelques-uns? Plus encore, les programmes du lycée doivent-ils être conçus pour assurer l'auto-reproduction des futurs professeurs de français?

Or la question des exercices où se réinvestit le corpus consacré est évidemment, la question vraiment névralgique. Elle constitue le point où se fait le passage d'une valeur d'usage (un plaisir pris à la lecture) en une valeur d'échange (enseigner des textes comme des modèles pour certaines pratiques). Et l'enjeu ici est doublement capital. Capital pratique, puisque se décide ainsi qui enseignera (fera des éditions, fera des commentaires, fera des cours) sur quoi. Et capital symbolique, puisque par cette opération, ce qui est la valeur d'usage de certains (ceux qui ont pris du plaisir à lire tel ou tel auteur) se convertissant en valeur d'échange (une valeur qui s'impose dans la collectivité) se pose comme une valeur tout court, une valeur absolue. Ce que révèle la véhémence et le lexique tragique des querelles sur le sujet, c'est, notamment, qu'il y va des intérêts les plus concrets des belligérants de l'un et l'autre côté.

Des intérêts qui renvoient eux-mêmes, troisième question, à des enjeux sociaux - la reproduction ou la démocratisation - et plus largement politiques. On peut dire que d'un côté, il y va d'un usage à vocation identitaire et de l'autre, utilitaire, et que cette tension constitue bien un mouvement tectonique de longue durée. Ainsi, en 1738 le but majeur de d'olivet était de doter la France de « classiques comme l'avait déjà fait les Italiens ", mais de "classiques français» qui attestent que la France a réussi la translatio studii à proportion de la translatio imperii. De la littérature comme une des voies de l'impérialisme. Deux siècles et demi plus tard, les "Républicains » - le choix même de ce nom a du sens - s'inquiètent parce que les monuments de la culture laissent affleurer les ruines de l'empire. Ils écrivent (voir annexe a) : « la meilleure chance d'intégration [à] donner aux jeunes immigrés [était] de leur apprendre le latin et le grec pour qu'ils ouvrent les yeux sur l'unité de cette culture méditerranéenne qui est à la fois la leur et la nôtre ». Or sous 
le nom d' « immigrés » il s'agit en fait d'élèves qui, pour la plupart, sont nés en France, et qui ne sont pas tous issus de familles venues du Bassin Méditerranéen, et qui enfin, lorsque leur origine est Nord-Africaine, c'est une Afrique du Nord bien postérieure à l'influence romaine. De l'exaltation du prestige de la nation à la peur d'une perte de l'identité nationale, la tonalité a changé à proportion du changement dans les publics concernés en fonction des changements dans le rôle social de l'Ecole. À cet égard, les différences de contextes importent au plus haut point. Mais elles n'obèrent pas le fait que la littérature légitime apparaît dans les deux cas tributaire d'enjeux politiques, vouée à souder une communauté nationale, soit au nom de l'identité, soit au nom de l'utilité.

Voilà les trois questions de reconnaissance et de légitimité auxquelles, avec mes deux querelles, je souhaitais donner un peu de substance. Voici maintenant quatre réflexions qu'elles me semblent appeler.

Une première réflexion, que je crois très évidente, est qu'il peut être plus pertinent de parler de «légitimation» que de "légitimité». Pas seulement parce que, linguistiquement parlant, la «légitimité » n'est que le résultat d'un processus de légitimation, mais parce que ce processus apparaît comme un combat incessant. Si j'ai choisi ici deux querelles séparées par une longue durée c'est pour faire apparaître cette permanence. On pourrait dire alors que la légitimation est un combat permanent, et la légitimité un certain état des forces aux divers moments de ce combat. Je crois que cela est, disais-je, évident, et on pourrait le théoriser par une formulation inspirée par les travaux de Pierre Bourdieu, comme le résume la formule selon laquelle le champ littéraire est tissé par des luttes pour le «monopole de la légitimité littéraire, c'est-à-dire pour conquérir le pouvoir d'énoncer la définition légitime de la littérature légitime (Bourdieu, 1992 : 311).

Mais ici, sommes-nous exactement dans le champ littéraire? Alors ma deuxième réflexion pourrait être un moyen de nuancer la première. Elle prendra appui sur les travaux du groupe de recherches Agon qui étudie les «querelles, disputes et controverses", tant dans les domaines littéraire que scientifique, religieux ou philosophique, ce qui conduit à s'interroger sur les similitudes et différences entre les composantes du champ culturel et entre les périodes de son histoire. Ici, les différences de contextes historiques prennent toute leur importance. Ainsi, au milieu du XVIII ${ }^{\mathrm{e}}$ siècle, quand l'enseignement des Lettres françaises en est à ses balbutiements et ne s'adresse qu'à un public restreint, les débats participent de la logique du champ littéraire. En atteste le fait que l'instance vers laquelle se tournent les belligérants est l'Académie, institution littéraire par excellence. À l'orée $\mathrm{du} \mathrm{xxI}^{\mathrm{e}}$ siècle en revanche, alors que la démocratisation a appelé dans les lycées une population nouvelle, le conflit relève du champ de l'Ecole, et l'institution de référence devient le ministère de l'Education. Ainsi, les lieux de consécration et de constitution de la légitimité littéraire se sont déplacés. On connaît ceux du champ littéraire, tels que les prix et les académies. Mais le champ scolaire ne retraduit pas directement les formes de consécration acquise dans le champ littéraire. Il les renforce, les fait passer de la consécration à la canonisation. Mais il les retraduit aussi dans sa logique propre. Surtout en France. Et c'est ce que soulignent les travaux comparatistes d'Agon. Ils suggèrent en effet l'hypothèse que la France est peutêtre le pays de ces querelles par excellence. Elles s'y répètent en effet avec une fréquence 
qui ne semble pas avoir d'équivalent ailleurs en Europe. Le caractère très national, étatisé et centralisé des institutions, vrai pour l'Académie mais plus sensible encore pour l'Ecole, fait que la question des classiques y est devenue plus qu'ailleurs une affaire d'Etat. Il y a là pour une part un phénomène très général, et pour une autre part une spécificité française. Le phénomène universel pourrait se décrire autour d'une formule de Louis Marin qui disait que la force se fait pouvoir lorsqu'elle parle, lorsqu'elle tient un discours qui légitime sa domination. Et la spécificité française, dans le fait que les pouvoirs y ont énormément utilisé la référence culturelle, et en particulier la référence littéraire, dans leur discours de légitimation et comme moyen de définition d'une "identité française ", et qu'en retour les institutions étatiques ont pris une place prépondérante dans la définition de la culture légitime. D'où la violence des querelles françaises à ce sujet spécificité qui n'est pas identique dans d'autres domaines. Et d'où aussi, pour un Français, la tentation de voir toutes les querelles comme des conflits de légitimation et le risque de radicaliser ainsi à l'excès; après tout, la polémologie est peut-être bien une spécialité française. Je laisse la question ouverte.

Mais s'interroger sur les contextes conduit aussi à s'interroger, troisième réflexion, sur les situations de conflits. Si je reviens un instant à mes exemples, ceux d'entre vous qui connaissaient l'abbé Desfontaines le connaissaient peut-être à travers le long conflit qu'il a eu avec Voltaire, et sa querelle avec d'Olivet, lui-même lié à Voltaire, peut et doit se lire aussi dans ce contexte. On pourrait faire de même une analyse des chaines de conflits dans lesquelles a pris place la querelle des programmes en l'an 2000, mais j'abrège. Pour en venir à cette suggestion, en prenant appui sur des travaux comme ceux du Groupe de Recherche en Sociologie Pragmatique que les querelles sur la légitimation culturelle prennent place et sens dans des chaines d'affrontements plus larges, des affrontements qui portent sur des enjeux qu'elles retraduisent à leur façon mais qui les dépassent. De fait, il est manifeste que dans de telles affaires, il y a bien pour une part des affrontements d'intérêts très pratiques, tout ensemble matériels et symboliques. Si l'on crée les classiques, qui aura le prestige et les revenus d'en réaliser les éditions? Ou bien, en l'an 2000, voyez par exemple dans l'appel du 4 mars comment les professeurs de langues anciennes s'inquiètent devant des possibles fermetures de classes et des auteurs et directeurs de certaines collections devant la baisse de leurs ventes et de leur audience. Mais il y va aussi d'intérêts d'un autre ordre. Des intérêts politiques tels que le prestige de la France, l'identité nationale ou encore la reproduction sociale. Mais tout cela cristallise autour d'une autre sorte d'intérêt.

21 Laquelle me paraît mériter une ultime réflexion. On a vu tout à l'heure que le corpus canonique était toujours apparemment consensuel et jamais vraiment défini. Du coup, ne sont jamais définies non plus la valeur sur laquelle il est censé se fonder. Ses possibles valeurs d'échange sont exprimées dans les exercices auquel il donne lieu. Mais ses valeurs d'usage? Paradoxe de la légitimité littéraire : elle revendique comme critère une valeur supérieure dont elle ne peut jamais dire en quoi elle consiste. Question à la fois névralgique et sans issue, on le sait bien quand on travaille sur des objets culturels, donc des objets qui relèvent des jugements de goût et non de critères qui pourraient se fonder sur l'épreuve scientifique du réel, qui n'a pas de vrai et de faux, mais des vérités toujours relatives. Et c'est cette forme relative que notre sujet nous invite à interroger.

On pourrait ici l'envisager sous la forme de trois scénarios - ou scenarii si l'on préfère, au nom du purisme. 

consisterait à considérer la culture légitime, comme un réservoir où des forces et groupes divers peuvent puiser de façons diverses selon leurs besoins propres, qu'ils hiérarchisent les contenus légitimés en fonction de leurs besoins et que les querelles révèlent et ces hiérarchies et ces besoins. Ce serait, si l'on veut, un scénario de la légitimation relative. Mais l'on a vu que les jeux sur l'implicite du canon littéraire étaient en fait des armes. On pourrait donc aller encore un peu plus loin. Ceux qui manient ces implicites exercent d'un pouvoir sur ceux qui ne jouissent pas des mêmes connivences. Par-delà leurs querelles, ils tracent ainsi les frontières d'un espace de pouvoir. On pourrait ici s'inspirer de l'idée d'hégémonie selon Gramsci. Ce qui conduirait à un troisième scénario, qu'on 
pourrait appeler celui de la relativité absolue: les débats sur la littérature légitime révèlent en fait des espaces dans lesquels s'affrontent des forces sociales et politiques qui définissent chacune la légitimité culturelle en fonction de leurs besoins socio-politiques. Qui, en ce cas, ne font pas que puiser dans un réservoir, mais décident de la valeur des textes et des œuvres et donc de l'existence même du réservoir, selon des critères entièrement externes. Les autodafés des pouvoirs totalitaires, qu'ils soient politiques ou religieux, imposent de prendre en compte une telle hypothèse.

Entre ces trois scénarios, sans prononcer mon choix faute de disposer d'assez d'espace pour en légitimer un plus que les autres, je constate qu'on ne peut les évoquer sans revenir sur la question de la valeur, c'est-à-dire des formes d'intérêt. Reconnaître de la légitimité à certains objets littéraires n'est-ce pas en fait désigner l'importance de certaines questions auxquelles ils nous renvoient ? Et si- je dis bien « et si » car je me réfère ici à mes propres essais de théorie (Viala, 2005, Viala, 2009) - la littérature était un creuset qui s'échauffe les intérêts de curiosité et de plaisir qui, d'abord échauffés pour servir des enjeux pratiques, idéologiques et politiques, restent ensuite, par sa qualité propre de communication différée fondée sur ces intérêts-là, disponible pour d'autres usages.

Alors peut-être qu'entre les scénarios ou scénarii que je dessinais tout à l'heure, il faudrait en prendre non pas un seul, non pas non plus trois, mais bien deux à la fois, et en ce cas, sans doute les deux derniers.

\section{BIBLIOGRAPHIE}

BOURDIEU, Pierre (1992). Les Règles de l'art. Paris : Seuil.

FINKIELKRAUT, Alain (2006). Ce Que Peut La Littérature. Paris : Stock.

FINKIELKRAUT, Alain (2007). La Querelle de l'Ecole. Paris : Gallimard.

JARRETY, Michel (dir.) (2000). Propositions pour les enseignements littéraires. Paris : PUF.

JARRETY, Michel (dir.) (2001). « L'avenir d'un passé », Europe, nº 863.

JOSTE, Agnès (2002). La Réforme du français au lycée, contre-expertise d'une trahison. Paris : Mille et une nuits.

LECHERBONNIER, Bernard (2005). Pourquoi veulent-ils tuer le français? Paris : Albin Michel.

MARX, William (2006). L'Adieu à la littérature. Paris : Minuit.

(2000 ; 2005). Revue L'Ecole des Lettres, numéros spéciaux de mars et mai ; et nº 9.

(2001). Revue Pratiques, notamment n 109-110, juin.

(2005). Revue Le Débat, n 135, mai-août.

(2001). Sauver les Lettres (collectif), Des professeurs accusent. Paris : Textuel.

TODOROV, Tzevan (2007). La Littérature en péril. Paris : Flammarion. 
VIALA, Alain (1985). Naissance de l'écrivain. Sociologie de la littérature à l'âge classique. Paris : Minuit.

VIALA, Alain (2005). Lettre à Rousseau sur l'intérêt littéraire

Paris : PUF, collection « Quadrige/Essais \& Débats ».

VIALA, Alain (2009). La Culture littéraire, Paris : PUF.

\section{ANNEXES}

\section{Extraits des textes de mars 2000}

\section{a. C'est la littérature qu'on assassine rue de Grenelle (Le Monde, 4 mars 2000)}

(...) C'est tout un pan de notre culture qu'on met à bas, méthodiquement, sans crier gare. Proust en prend pour son grade, lui qui croyait, l'infortuné, que « la vraie vie, la seule vie réellement vécue, c'est la littérature ».

La littérature fut insensiblement dissoute dans l'eau tiède de la "para-littérature ", production légère à la mode du jour, superficielle et hâtivement écrite, mais prête à consommer : plus besoin d'ennuyer les élèves avec les subtilités lexicales ou syntaxiques de La Fontaine ou de La Bruyère. Les repères chancelaient, mais les professeurs prenaient encore largement liberté de faire aimer les grands textes qu'on avait su leur faire aimer. On pouvait créditer les ministres d'une intention louable, ou d'une bonne excuse, puisque ces naïfs croyaient qu'il était nécessaire de s'adapter au nombre croissant des élèves dont certains n'étaient guère familiers d'une culture à laquelle leurs familles n'avaient pas pu les préparer. Nous savons aujourd'hui quelle sottise ce fut, et qu'une telle politique allait à rebours de cette égalité des chances (...).

Nous savons en effet que, si la littérature ancienne et moderne disparaît de l'enseignement offert à tous, elle survivra comme un rare privilège et une supériorité sociale dans les familles les plus aisées. Un privilège, parce que seuls les jeunes gens les plus favorisés sauront le plaisir et le surplus d'être que l'on peut tirer de la culture littéraire. Une supériorité sociale, parce que seuls ces jeunes gens auront appris à écrire, à penser et parler avec maîtrise, souplesse et efficacité, quand les autres, plus fragiles, ne sauront vraiment s'affirmer dans leur vie ni leur profession et, faute d'un jugement patiemment aiguisé, seront, comme citoyens, les gobe-mouches des « Temples solaires » à venir.

On supprime la dissertation. Mais (...) il s'agit d'apprendre à écrire, à penser et à construire sa pensée pour la transmettre dans toute la subtilité de ses nuances, sans méprises ni à-peu-près, c'est-à-dire de se préparer à tous les métiers où, oralement comme par écrit, c'est de s'exprimer au mieux qu'il s'agit chaque jour.

N'alléguons pas Péguy, dont la mère rempaillait des chaises, qui entra rue d'Ulm avant de devenir écrivain, et qui sans la formation de cette école-là ne fût pas - songeons-y un instant - devenu écrivain, ni Camus, aussi pauvre et aussi démuni et qui doit se retourner dans sa tombe, ni tant d'autres encore. Mais enfin, c'est de cela qu'il s'agit.

Les petits Péguy d'aujourd'hui sont bien souvent des beurs : quelle plus grande preuve d'estime, d'intérêt et de respect pourrions-nous leur manifester que de leur donner la chance de la culture, au lieu de leur claquer la porte au nez en décrétant : «Pas de 
littérature pour vous!» Quelle meilleure chance d'intégration donner aux jeunes immigrés que de leur apprendre le latin et le grec pour qu'ils ouvrent les yeux sur l'unité de cette culture méditerranéenne qui est à la fois la leur et la nôtre ? Tout à l'inverse, par cette mesquinerie d'agent comptable qui ne voit pas plus loin que le bout de son crayon, on décrète qu'on n'ouvrira pas de classe de latin ou de grec s'il n'y a pas au moins quinze élèves pour y entrer. Entendez bien : cela coûte trop cher. (...) A-t-on seulement tiré la leçon des difficultés que connaissent les Presses universitaires de France? La vente des essais qui, par priorité, s'adressent aux étudiants, que leurs professeurs écrivent pour eux, a baissé de $40 \%$ depuis dix ans. Pourquoi ? Parce que ces livres qui étaient censés leur apporter le savoir nécessaire à leur futur métier, ces livres sont devenus trop difficiles pour l'immense majorité d'entre eux. Non que les étudiants d'aujourd'hui soient plus sots que nous n'étions, mais parce que, en arrivant à l'université, ils n'ont plus derrière eux la solide préparation qui nous avait déjà formés. (...)

C'est la chronique d'une mort assurée et d'une mort préméditée. (...) »

(Texte signé par un groupe d'universitaires)

\section{b. Contre la suppression de la dissertation (Ibid.)}

« La dissertation, cette " vieille dissertation », est " moribonde », nous dit-on. Au nom de l'innovation, du Xxi ${ }^{\mathrm{e}}$ siècle, du nouveau public lycéen, on nous annonce discrètement (en plein été) la suppression de la dissertation au baccalauréat de français 2002 : le nouveau programme de français en seconde, tel qu'il est présenté (Bulletin officiel de l'éducation nationale hors-série no 6 du 12 août 1999), ne laisse guère planer de doute à ce sujet, il laisse transparaître des présupposés condamnables et des enjeux alarmants (...) L'enseignement du français au lycée subit une révolution inacceptable, guidée par des objectifs aussi ambitieux sur le papier que matériellement irréalisables.(...) ». (Texte signé par deux professeurs de lycée)

\section{c. Comment sauver la littérature ? (Le Monde, 8 mars 2000)}

« Nous pouvons reconnaître [dans les deux articles cités] des convictions dont on peut penser qu'elles constituent le plus petit dénominateur commun pour la grande majorité d'entre nous La première de ces convictions est que l'enseignement de la littérature joue un rôle central dans notre discipline ; la seconde est que ce dernier a une mission capitale dans une perspective de démocratisation véritable de l'école, dont tout le monde sait qu'elle ne se réduit pas à une massification. Enfin les deux textes soulignent la nécessité pour les lycéens « d'apprendre à écrire, à penser et à construire [leur] pensée ». Voilà un triangle de certitudes sur lequel on pourrait espérer construire un large consensus... Mais hélas ! Celui-ci cesse vite dès qu'on aborde la question de savoir ce qu'on met sous le mot: littérature (...) Il faut partir du constat de l'extrême diversité du public auquel un même programme d'enseignement est destiné : diversité sociale, culturelle et ethnique, extrême disparité des espoirs que les jeunes placent dans l'école, de l'adhésion qu'ils manifestent spontanément, au travers des filtres de la culture familiale ou du groupe, aux savoirs qu'on leur propose. Cela ne signifie certes pas qu'il faille concevoir des programmes divers pour des élèves divers, mais que les ambitions des programmes doivent être telles qu'elles puissent s'appliquer aux situations d'enseignement les plus variées. (...) Si l'on veut servir la cause de la littérature, il faut d'abord faire découvrir aux apprentis lecteurs de tous âges qu'il y a des livres qui peuvent les intéresser, les émouvoir, les informer, 
stimuler leur pensée.

Les propositions doivent donc être très ouvertes. On ne peut plus aujourd'hui s'enfermer dans le champ de la seule littérature française, ce qui ne signifie pas qu'il ne faut pas lui faire une place de choix. La légitimité du recours aux littératures contemporaines, aux littératures en train de se faire, doit de même être affirmée. C'est dans ce contexte d'un corpus littéraire considérablement élargi qu'il convient de penser la découverte de la « littérature du patrimoine ». Qu'on nous comprenne bien : il ne s'agit pas de faire à cette littérature une place mineure, mais au contraire de la sortir du ghetto où son statut scolaire l'enferme trop souvent. (...) Doit-on définir le programme d'apprentissage culturel de toute une classe d'âge en fonction des perspectives de spécialisation de quelques-uns ? Plus encore, les programmes du lycée doivent-ils être conçus pour assurer l'auto-reproduction des futurs professeurs de français? (...) On est donc tenté pour conclure de renverser l'affirmation que formule le titre de l'un des articles : c'est peutêtre l'enseignement actuel de la littérature qui contribue à l'assassinat de celle-ci. » (Texte signé par le Bureau de l'Association Française des enseignants de Français)

\section{NOTES}

1. À Paris, chez Gandouin; l'approbation est datée du 10 mars 1738. L'ouvrage est dédié à l'Académie. D'Olivet souhaite que «La France ait ses classiques aussi bien que l'Italie» (p.1) et souligne qu'« ils peuvent servir de modèles et pour bien penser et pour bien écrire ». Pour cela, il faut de lancer une série d'éditions critiques des « ouvrages dont le mérite est depuis cinquante ou soixante ans avoué de tout le monde » (p.5) et propose que la liste en soit dressée par l'Académie. Pour sa part, il se propose à cet égard de relever les "petites fautes de style que Racine a pu commettre »; il exclut de son corpus La Thébaïde, parce qu'elle n'est que «l'essai d'un jeune homme » (p. 6).

2. L'Abbé Desfontaines ouvre les hostilités dans la livraison de mai 1738 de son périodique Observations sur les écrits modernes (s.e., n.p.). D'Olivet fait appel au soutien de son confrère à l'Académie Française, le Président Bouhier dans une Lettre à $M$ le président Bouhier, Paris, Gandouin, (s.d., i.e.1738). Ensuite, Desfontaines publie son Racine vengé, ou examen des remarques des remarques de M. l'Abbé d'Olivet sur les cuvres de Racine, Avignon, s.n., 1738 (une note mss de l'exemplaire de La BNF indique que c'est chez la «Veuve Tissot»). L'ouvrage s'ouvre par une épître dédicatoire «À l'Académie Françoise » où Desfontaines fait l'Académie « juge du différend littéraire qui est entre M. d'Olivet et [lui]» (p.iiii). Il fait l'éloge de (p. iii) « Racine et Despréaux, ces pères du Parnasse français, deux grands modèles dont l'autorité fait loi en matière de versification ». Mais il constate que les jeunes gens ont besoin d'écrire en prose, et met d'Olivet au défi de faire le même travail sur « les Pellisson, les Bussy, les Bouhours, les Fléchier, les Fleury, les Vertot ». Il estime (p. iii) que Racine fait quelques «fautes de pensée mais très peu de fautes de langue " parce qu'il use de la langue poétique et que donc quand d'olivet le "corrige », il produit un « ouvrage très dangereux pour les Lettres et en particulier pour la poésie française ». Viennent ensuite les textes d'une Lettre de J.-B. Rousseau (de Bruxelles, le 16 novembre) et de la réponse de Desfontaines (de Paris, le 20 novembre), avec notamment (p. vii) : "Quand on a l'esprit bien fait ne se prête-t-on pas en galant homme à de petites railleries innocentes qui n'excèdent point les bornes d'une dispute littéraire ", et (p.viii) « l'ouvrage polémique que je vais donner au public était nécessaire pour la conservation des précieuses libertés du Parnasse français ». Ensuite, il suit les Remarques de D'Olivet pendant 151 p., avec à la fin une discussion sur la tragédie en prose, incluant des citations de Bouhier et critiquant l'Oedipe en prose de MDLM ( = Monsieur de La Motte, 1730) parce qu'il n'y a « point de poésie sans versification ». 
3. Je renvoie ici aux données élaborées par le programme de Recherche AGON : Disputes et Querelles dans la première modernité ; voir le site www.agon.paris-sorbonne.fr/en

4. Ed. cit., p. 5.

5. Naissance de l'écrivain. Sociologie de la littérature à l'âge classique, Paris, Minuit, 1985.

6. En plus de ces deux textes, ont paru dans le journal Le Monde des tribunes libres d'A. Boissinot, le 9 mars 2000, et de Ph. Hamon, le 16 mars; puis, notamment encore, et par ordre alphabétique pour ne pas surcharger de détails) : Académie française, «Déclaration de l'Académie sur le recul des études littéraires", le 6 avril 2000, sur le site internet de l'Académie; A. Finkielkraut, diverses émissions Répliques sur France Culture, 2002-2005, partiellement reprises dans Que peut la littérature ?, Paris, Stock, 2006 et La Querelle de l'Ecole, Paris, Gallimard, 2007 ; M. Jarrety (dir. de), Propositions pour les enseignements littéraires, Paris, PUF, 2000 (actes du meeting tenu à la Sorbonne le 16 mai 2000), et «L'avenir d'un passé ", Europe, $\mathrm{n}^{\circ}$ 863, 2001 ; A. Joste, La réforme du français au lycée, contre-expertise d'une trahison, Paris, Mille et une nuits, 2002 ; B. Lecherbonnier, Pourquoi veulent-ils tuer le français ?, Paris, Albin Michel, 2005 ; W. Marx, L'Adieu à la littérature, Paris, Minuit, 2006 ; Revue L'Ecole des Lettres, notamment numéros spéciaux de mars et mai 2000 et n 9 de 2005, Revue Pratiques, notamment n 109-110, juin 2001 ; Revue Le débat, n 135, mai-août 2005 ; Sauver les Lettres (collectif), Des Professeurs accusent, Paris, Textuel, 2001. T. Todorov, La Littérature en péril, Paris, Flammarion, 2007. NB : je n'ai pas inclus les ouvrages de deux ministres, C. Allègre et $\mathrm{X}$. Darcos, qui ont eu en charge l'Education Nationale, et qui insèrent de telles questions dans leurs polémiques sur l'Ecole.

\section{RÉSUMÉS}

Si l'on essaye de baliser l'histoire du canon littéraire français, deux querelles semblent permettre de repérer les enjeux de la légitimation des auteurs nationaux par leur érection en " classiques ». L'une, le première en date, a vu au premier tiers du XviII siècle, des érudits s'affronter non tant sur la liste des «classiques » qu'il s'agissait alors d'inventer que sur les usages qui pouvaient en être faits. L'autre, la plus récente en date, advenue autour des programmes d'enseignement en 2000, apparaît elle aussi comme un débat sur les usages de la littérature légitime. Et par-delà ces usages, ces débats engagent aussi des intérêts de deux ordres : corporatifs d'un côté, politiques de l'autre. Le propos n'est pas alors de prendre parti en ces matières que de faire ressortir comment la question de la légitimation est en fait une question non de valeur en soi mais de valeurs sociales.

History of the the French literary canon can be analyzed from two particular dispites. The first one at the origin of this canon was held in 1738 between erudites who were trying to elaborate and publish a list of canonical French writers. The other one, the most recent at date, was happened en 2000 about national programmes for High Schools. 1738 showed that the main concern was not to decide who are the authors to be legitimated but the ways of use of Literature. Similarly, on 2000, the matter was the question of the reasons to teach legitimated works, from professional and politics points of view. Therefore, what happens here is that the criteria to define what is legitimated are social rather than purely esthetical. 
INDEX

Mots-clés : légitimation, école, institution littéraire, valeur

Keywords : legitimation, school, literary institution, value

\section{AUTEUR}

ALAIN VIALA

Un. d'Oxford /Un. de Paris iii

alain.viala[at]lmh.ox.ac.uk 